\title{
Treatment results and safety assessment of the LARS system for the reconstruction of the anterior cruciate ligament
}

\author{
Maciej Kentel ${ }^{1, A, C, E, F}$, Michał Barnaś ${ }^{1, A-F}$, Jarosław Witkowski ${ }^{2, E, F}$, Paweł Reichert ${ }^{3, A, D-F}$ \\ ${ }^{1}$ Orthopedics and Traumatology Department, eMKa MED Medical Centre, Wrocław, Poland \\ 2 Division of Sports Medicine, Department of Physiotherapy, Faculty of Health Sciences, Wroclaw Medical University, Poland \\ ${ }^{3}$ Department of Trauma and Hand Surgery, Faculty of Medicine, Wroclaw Medical University, Poland \\ A - research concept and design; B - collection and/or assembly of data; C - data analysis and interpretation; \\ $D$ - writing the article; $E$ - critical revision of the article; $F$ - final approval of the article
}

\section{Address for correspondence}

Pawel Reichert

E-mail: pawelreichert74@gmail.com

Funding sources

None declared

Conflict of interest

None declared

Received on December 23, 2020

Reviewed on December 24, 2020

Accepted on December 30, 2020

Published online on April 28, 2021

Cite as

Kentel M, Barnaś M, Witkowski J, Reichert P. Treatment results and safety assessment of the LARS system for the reconstruction of the anterior cruciate ligament. Adv Clin Exp Med. 2021;30(4):379-386.

doi:10.17219/acem/132037

DOI

10.17219/acem/132037

Copyright

Copyright by Author(s)

This is an article distributed under the terms of the

Creative Commons Attribution 3.0 Unported (CC BY 3.0)

(https://creativecommons.org/licenses/by/3.0/)

\begin{abstract}
Background. Anterior cruciate ligament $(\mathrm{ACL})$ reconstruction is the prevailing procedure in cases of $\mathrm{ACL}$ rupture.

Objectives. To assess the long-term safety of implementing a synthetic ligament with the Ligament Advanced Reinforcement System (LARS) in primary reconstruction of the ACL.

Materials and methods. The retrospective analysis involved 403 patients who had undergone ACL reconstruction with the same results in clinical and functional assessments. The patients comprised 2 groups. In group I, a LARS graft was implemented, while in group II, an autograft was used. The Lachman test, anterior drawer test, pivot-shift test, Lysholm scale, IKDC 2000, pain posited to be experienced, the possibility of postoperative complications, the time required to return to work, and revision surgery were all considered and analyzed.
\end{abstract}

Results. The visual analogue scale (VAS) pain score in group I ranged from $37.34 \pm 8.22 \mathrm{~mm}$ on day 3 to $17.21 \pm 5.45 \mathrm{~mm}$ on day 28 . In group $\|$, it ranged from $64.72 \pm 10.20 \mathrm{~mm}$ on day $3(p<0.05)$ to $18.67 \pm 6.57 \mathrm{~mm}$ on day 28 . The period of time taken to return to office work in group I was $7.04 \pm 1.82$ weeks, and $9.21 \pm 1.75$ weeks in group $\|(p<0.05)$. The time taken to return to physical work in group I was $20.50 \pm 2.91$ weeks, and $21.12 \pm 3.12$ weeks in group II. Postoperative scar and local complications were statistically less prominent in group I. The cost and number of revision surgeries were greater in the first group.

Conclusions. Reconstruction of the ACL using a synthetic graft such as $L A R S$ yields similar results to an autograft in a cohort follow-up. Long-term results show a large number of revision surgeries when LARS is used. This method should be used with caution.

Key words: ACL reconstruction, LARS graft, arthroscopy, synthetic graft, autograft 


\section{Background}

The anterior cruciate ligament (ACL) is recognized as the most regularly injured ligament in the human knee. ${ }^{1}$ In the USA, the average frequency of ACL injury incidence numbers 200,000 cases per year. ${ }^{2}$ The injury most commonly occurs in individuals who are physically active and engage in dynamic sports. ${ }^{1}$ For patients seeking to return to such physical activity, the standard treatment for ACL injury is ligament reconstruction, ${ }^{3}$ followed by a postoperative physiotherapeutic procedure. ${ }^{4,5}$ Restoring knee stability is the goal of reconstruction, and in turn aims to reduce the risk of secondary injuries such as further damage to the menisci and degenerative osteoarthritis. ${ }^{6,7}$ An analysis of epidemiological data points to an increase in the incidence of ACL ruptures and ensuing reconstructions. ${ }^{8}$ During the reconstruction, the torn ligament can usually be replaced with an autograft or allograft. Synthetic ligament usage is also a possible graft option. Autograft choices are the patellar, hamstring and quadriceps tendons, while the allograft options consist of the quadriceps, patellar, Achilles, hamstrings, anterior and posterior tibialis tendons, and the fascia lata. ${ }^{9-11}$ Truncated surgical and anesthesia time, fewer postoperative complications, lower chance of morbidity at the harvest site, quicker postoperative recovery, less incidence of postoperative arthrofibrosis, and a lower amount of postoperative pain experienced are among the main advantages of allograft usage for ACL replacement. ${ }^{4,5}$ In contrast, autograft usage may lead to higher rates of re-rupture, have limited availability, delay the process of healing and ligamentization when compared to autografts, have a higher risk of transmitting disease, and result in greater overall costs. ${ }^{7,12,13}$ The synthetic materials being used in ACL reconstruction were introduced in hope of improving the firmness and stability of the graft after reconstruction, reducing instances of donor site morbidity, and eliminating the potential for disease transmission. ${ }^{14}$ That being said, the first artificial grafts were plagued by high rates of failure and synovitis reactivation, ${ }^{15}$ thus, with constantly advancing technology, new synthetic materials have been and are being developed for ACL reconstruction grafts. ${ }^{16}$ The emergence of synthetic materials in ACL deficient knee treatment came at the beginning of the $20^{\text {th }}$ century with the usage of silk and silver fibers. New synthetic materials being proposed for torn ACL replacements proliferated in the second half of the $20^{\text {th }}$ century. These materials include Supramid ${ }^{\circledR}$, Teflon $^{\circledR}$ or Dacron ${ }^{\circledR}$, Proplast ${ }^{\circledR}$, carbon fiber graft, ABC graft, Kennedy-LAD ${ }^{\circledR}$, Trevia, Leeds-Keio, Gore-Tex ${ }^{\circledR}$, PDS $^{\circledR}$, EULIT $^{\circledR}$, and Polyflex ${ }^{\circledR}$, Ligament Advanced Reinforcement System, and Ligament Advanced Reinforcement System (LARS) ${ }^{\circledR} \cdot{ }^{17,18}$ Artificial ligaments have piqued surgeons' interest for all these years because of the optimism for grafts that are easily available and stronger than soft tissue "off-the-shelf" grafts, simplification of surgery, and avoidance of graft harvesting and donor site morbidity.
However, most of the artificial grafts have been beset with high failure rates. One of the very few synthetic grafts gaining widespread popularity has been LARS ${ }^{\circledR}$. It is suggested, however, that the ligament not be considered a potential graft for primary reconstruction of the ACL, and should be treated as an alternative graft in special cases, so the optimal synthetic graft material remains controversial. ${ }^{19-21}$

\section{Objectives}

The aim of this study was to assess the long-term safety of implementing the LARS synthetic ligament in the primary reconstruction of the ACL.

\section{Materials and methods}

The reported study was a retrospective cohort study. The sample was composed of patients who had undergone primary ACL reconstruction at the Trauma and Orthopedics Department of eMKaMED Hospital in Wrocław, Poland. Out of the 572 male patients treated from 2012 to 2018 with a partial lesion of the ACL involving anteromedial bundle damage requiring primary unilateral intra-articular ACL reconstruction, 403 patients homogeneous in terms of age and gender met the inclusion criteria for the study. Group I consisted of 46 patients aged $33.2 \pm 10.6$ years who underwent primary unilateral ACL reconstruction of the knee using LARS graft; group II consisted of 357 patients aged $29.4 \pm 16.5$ years who underwent primary unilateral ACL reconstruction of the knee with the use of autograft.

The study was carried out in accordance with the principles of the Declaration of Helsinki and was given approval by the Bioethics Committee of the Wroclaw Medical University. Each participant was informed of the aim of the study and the applied approach, and each signed to indicate their informed consent for participation in the study. An additional goal of the evaluation was to present the patient with the technique that was to be applied. The ACL capacity was analyzed with the 3 tests that are most frequently applied in clinical practice. The orthopedic assessment dealt with the range of motion (ROM) in the knee joint and included the Lachman test, anterior drawer test and pivot-shift test. The analysis was combined with a subjective evaluation using the International Knee Documentation Committee (IKDC) 2000 and Lysholm scales. Special attention was focused on complication and revision surgery. The follow-up was carried out over the course of 3 years.

The first limb assessed was the non-traumatic limb in order to evaluate its ROM and stability, and to make the patient familiar with the technique of the examination.

The examination was performed at 3, 7, 14 and 28 days post-surgery, and then at 12, 24 and 36 months post-surgery. 
The ROM measurement of the knee was taken bilaterally using a standard goniometer. The inter-limb difference in anterior tibial dislocation taken from the Lachman test and anterior drawer test was judged normal $(0 ; 0-2 \mathrm{~mm})$, nearly normal (1+; 3-5 mm), abnormal (2+; 6-10 $\mathrm{mm})$, or severely abnormal $(3+;>10 \mathrm{~mm}) .{ }^{22}$ Anterolateral rotational knee stability was evaluated manually utilizing the pivot-shift test. The pivot-shift test was deemed negative when, referencing the ligament examination section of the 2000 IKDC, the anterolateral rotational dislocation of the tibia relative to the femur was equal in both lower limbs and positive when the difference between the limbs was judged + (glide),++ (clunk) or +++ (gross). Moreover, early and late complications eventually requiring revision surgery were also analyzed.

\section{Surgical technique}

Each of the patients was operated on by the same team, employing the same surgical technique and autograft (hamstring; Fig. 1) or LARS (synthetic graft) (Fig. 2). The graft was secured using the Endobutton (SmithNephew, Warszawa, Poland) on the femur and the ComposiTCP30 interference screw (Biomet, Warszawa, Poland) on the tibia. It was constructed using the "outside-in" technique with the aimer device.

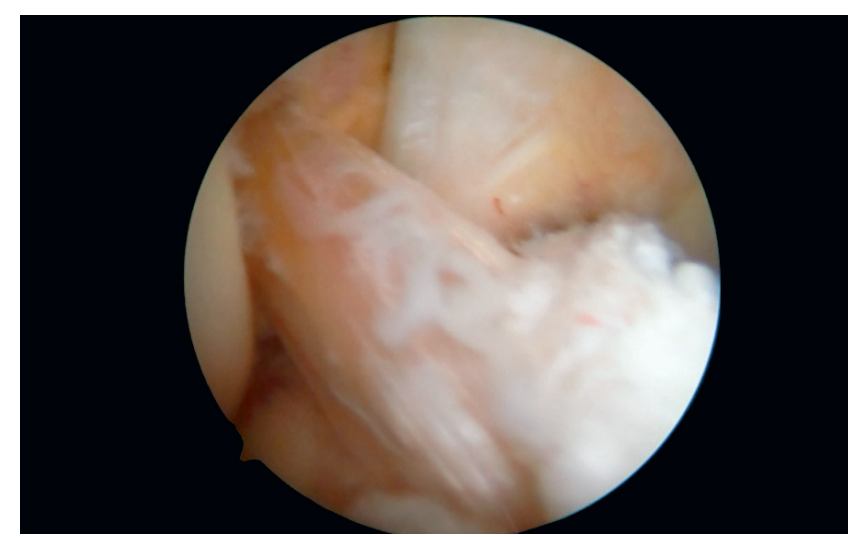

Fig. 1. Right knee $A C L$ reconstruction using autograft

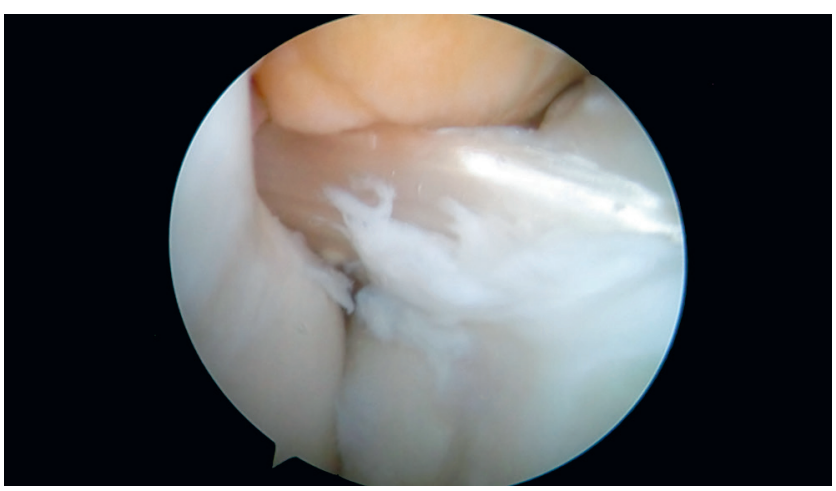

Fig. 2. Right knee ACL reconstruction using LARS

\section{Postoperative management}

Postoperative management was carried out according to ACL reconstruction protocol over a period of 6 months.

\section{Statistical analysis}

Statistical analysis was interpreted using TIBCO Statistica $^{\mathrm{TM}}$ (TIBCO Software Inc., Palo Alto, USA) and Microsoft Office Excel 365 Personal (Microsoft Corporation, Redmond, USA). As for characteristics of the sample and the survey of the knee "giving way", as it is colloquially referred to, the results of Lachman test, anterior drawer test and pivot-shift test were used and the number of patients (n) who obtained a given result in each group established. The arithmetic mean (AM) and standard deviation (SD) were calculated for the following parameters: active extension and flexion range in both the involved and the uninvolved leg $\left({ }^{\circ}\right)$, and the total scores obtained on the Lysholm scale (n points) and IKDC 2000 questionnaire. The Shapiro-Wilk test was conducted to determine the normality of distribution for the parameters. Statistical significance was set at $\mathrm{p}<0.05$.

The visual analogue scale (VAS) was applied on days 3, 7,14 , and 28 post-surgery (VAS is a scaled ruler from 0 to $100 \mathrm{~mm}$ with an accuracy of $1 \mathrm{~mm}$ ). Complications were then noted (hematomas at the collection site and intraarticular hematomas, skin sensation disturbances, pain in and around the back of the thigh). During the checkups at 12, 24 and 36 months post-surgery, complications were noted (skin sensation disturbance, pain in the back of the thigh).

\section{Choosing of the type of transplant by the patient}

After qualifying the patient for surgery on the basis of the abovementioned diagnostics and their acceptance of this method of treatment, the graft choice was explored with the patient. The ultimate decision as to the choice of graft, after the operating physician discussed the available options along with their benefits and drawbacks, was made by the patient.

\section{Results}

There was no statistical difference considering clinical examination and subjective and objective scales (i.e., Lachman test, anterior drawer test, pivot-shift test, Lysholm scale, and IKDC 2000 evaluation). Statistical differences were found when referring to return to work and to physical activity, and to resulting complications.

Patients after undergoing surgery utilizing LARS returned more quickly to work and physical activity. On the other hand, the main complications also concern 
the LARS group. Revision surgery was performed much more often in the LARS group than in the autograft group (50\% compared to $4.5 \%$ ). Moreover, revision operations in the LARS group were much more difficult to perform due to the greater damage to the knee.

\section{Functional assessment results}

Within 24 months of the reconstruction of the ACL, the average total number of points obtained on the Lysholm scale in group I was $98.65 \pm 4.25$ points. In group II, patients obtained an average of $97.42 \pm 4.28$ points in the same period after the surgery (Fig. 3). Comparative analyses of the results of the functional assessment based on the Lysholm scale did not show statistically significant differences in the examined groups $(\mathrm{p}=0.212)$.

Within 24 months of the reconstruction of the ACL, the average total number of points on the IKDC 2000 scale obtained in group I was $96.79 \pm 6.54$ points. In group II, patients obtained an average of $95.21 \pm 5.36$ points in the same period since surgery (Fig. 3). Comparative analyses of the results of the functional assessment did not show statistically significant differences in the examined groups $(\mathrm{p}=0.886)$.

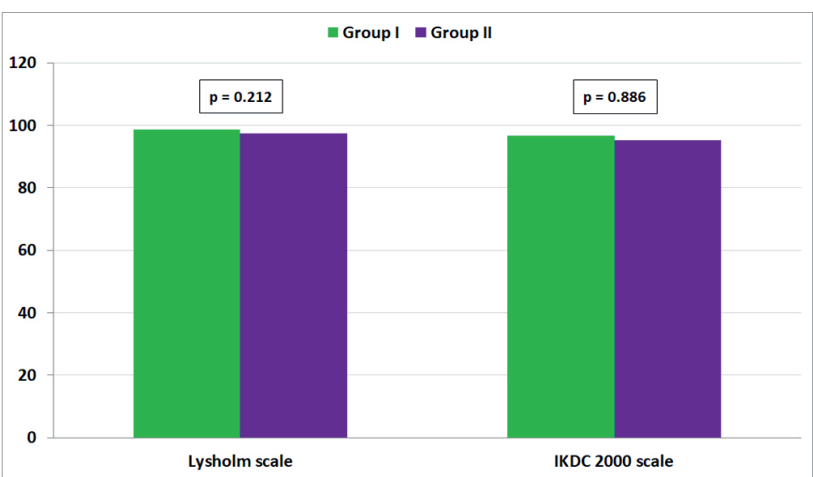

Fig. 3. Comparative analysis of the total number of points on the Lysholm scale and on the IKDC 2000 scale obtained in group I and group II

\section{Results of pain assessment}

The pain felt on a daily basis in the operated limb in group I can be interpreted as mild and at a maximum on the $3^{\text {rd }}$ day post-operation $(A M=37.34 \pm 8.22 \mathrm{~mm}$ ). A comparative inspection of the results of the assessment of the extremity of daily pain in the operated limb in group I exhibited statistically significant differences ( $\mathrm{p} \leq 0.001$ ) between the results acquired successively on postoperative days 3, 7, 14, and 28 (Fig. 4). The intensity of pain felt on a daily basis in the operated limb in group I was significantly lower ( $\mathrm{p} \leq 0.001)$ on postoperative day 7 $(\mathrm{AM}=29.22 \pm 7.67 \mathrm{~mm})$ than on postoperative day 3 $(\mathrm{AM}=37.34 \pm 8.22 \mathrm{~mm})$. Pain experienced also significantly decreased $(\mathrm{p} \leq 0.001)$ on the $14^{\text {th }}$ postoperative day $(\mathrm{AM}=20.67 \pm 6.66 \mathrm{~mm})$ compared to the $7^{\text {th }}$ postoperative day. The intensity of pain sensations in the operated limb on the $28^{\text {th }}$ postoperative day $(\mathrm{AM}=17.21 \pm 5.45 \mathrm{~mm})$ was comparable to the pain experienced on the $14^{\text {th }}$ day after surgery $(p=0.448)$. The pain experienced in the operated limb in group II on the $3^{\text {rd }}$ postoperative day was moderate in nature $(\mathrm{AM}=64.72 \pm 10.20 \mathrm{~mm})$. In the subsequent postoperative days, the values did not exceed $49.54 \mathrm{~mm}$, and can therefore be considered mild pain. Comparative analyses of the evaluation results of the intensity of pain experienced on a daily basis in the operated limb in group II, similarly to group I, showed statistically significant differences ( $\mathrm{p} \leq 0.001)$ between the results obtained successively on $3,7,14$, and 28 days post-surgery (Fig. 4). The intensity of daily pain in the operated limb in group II significantly $(\mathrm{p} \leq 0.001)$ decreased on day 7 after the surgery $(\mathrm{AM}=49.54 \pm 9.33 \mathrm{~mm})$ when compared to day 3 after surgery $(A M=64.72 \pm 10.20 \mathrm{~mm})$. Between days 7 and 14 post-surgery $(A M=32.42 \pm 8.20 \mathrm{~mm})$, the intensity of pain also decreased significantly ( $\mathrm{p} \leq 0.001)$. Pain felt in the operated limb on day 28 after the operation $(\mathrm{AM}=18.67 \pm 6.57 \mathrm{~mm})$ was significantly $(\mathrm{p} \leq 0.001)$ less intense than on the $14^{\text {th }}$ day after the operation. Comparing results of the assessment of the intensity of pain experienced daily in the operated limb showed that in group I, the level of pain was significantly lower than in group II (from $\mathrm{p} \leq 0.001$ to $\mathrm{p}=0.28$ ). A comparative analysis of the results obtained in both examined groups is shown in Fig. 4.

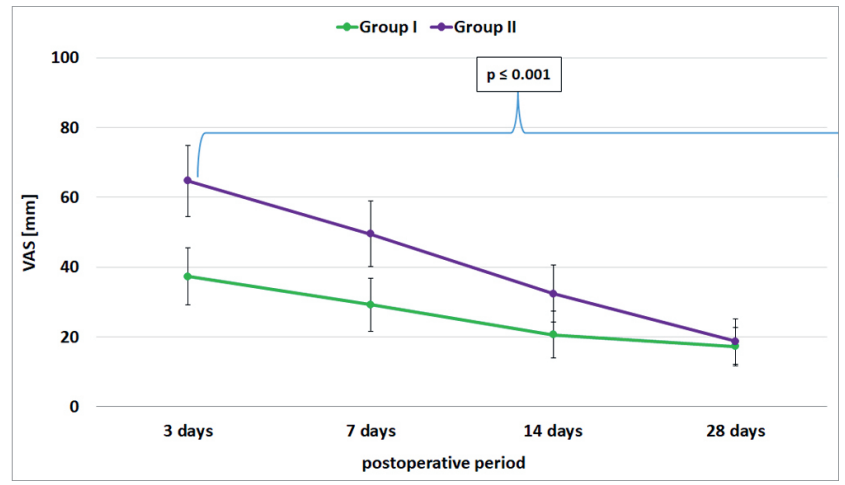

Fig. 4. Results of the assessment of the intensity of daily pain experienced in the operated limb in groups I and II on subsequent postoperative days

\section{Results of the return to work evaluation}

The patients from group I were able to return to office work significantly $(\mathrm{p} \leq 0.001)$ earlier $(\mathrm{AM}=7.04 \pm 1.82$ weeks $)$ than to physical work (AM = $20.50 \pm 2.91$ weeks). In group II, patients were also able to return to office work significantly ( $\mathrm{p} \leq 0.001)$ more quickly $(\mathrm{AM}=9.21 \pm 1.75$ weeks $)$ than to physical work (AM = 21.12 \pm 3.12 weeks) (Fig. 5). Patients from group I returned to office work significantly earlier $(\mathrm{p} \leq 0.001)$ than patients from group II. The time necessary in order to return to physical work was similar in both groups ( $\mathrm{p}=0.904)$ (Fig. 5). 


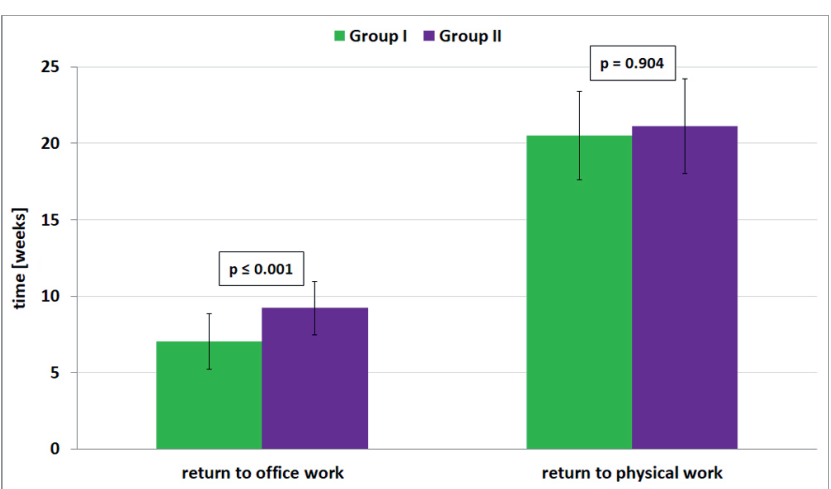

Fig. 5. Comparison of time taken to return to office work and to physical work in both groups

\section{Local postoperative complications}

Skin hypoesthesia was not present in any of the patients in group I. In contrast, it was present in 187 patients in group II, accounting for $52 \%$ of patients in this group. Symptoms of skin hypoesthesia subsided in less than 6 months after reconstruction of the ACL in 52\% of the patients. Symptoms of skin hypoesthesia subsided within 12 months of surgery in $6 \%$ of the patients. No patients in group I complained of discomfort in the posterior thigh of the operated limb. However, 84 patients from group II reported this symptom, which accounted for $24 \%$ of the patients from this group. Thigh pain resolved in less than 1 month of reconstruction of the ACL in 88\% of the patients. In the remaining $12 \%$ of patients, discomfort in the posterior thigh of the operated limb lingered for more than 12 months post-surgery. Twenty-four patients from group II had a hematoma at the graft site, which made up $6 \%$ of group II. Three out of 24 patients with hematoma received conservative treatment, and 1 patient out of the 4 with hematoma required surgical intervention.

\section{Late complications}

Revision surgery in autograft group was required in 21 cases $(5.6 \%)$ because of: secondary instability (loosening graft) -4 cases, secondary instability (graft rupture) -14 cases, arthrofibrosis -2 cases, and joint infection -1 case.

Revision surgery in LARS group was performed in 23 cases $(50 \%)$ because of: secondary instability (loosening graft) -1 case, secondary instability (graft rupture) - 18 cases, arthrofibrosis -2 cases, joint infection -1 case, synovitis -2 cases, cartilage lesion -2 case, and synovial cyst -5 cases (Fig. 6-11).

\section{Discussion}

The ACL reconstruction in adults using the LARS technique yields good results in short-term postoperative observations. The main advantages are: the possibility

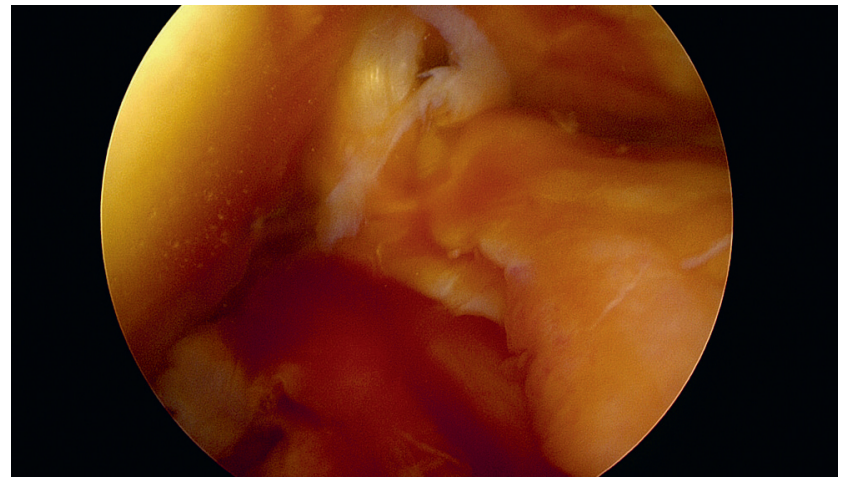

Fig. 6. LARS rupture after 12 months

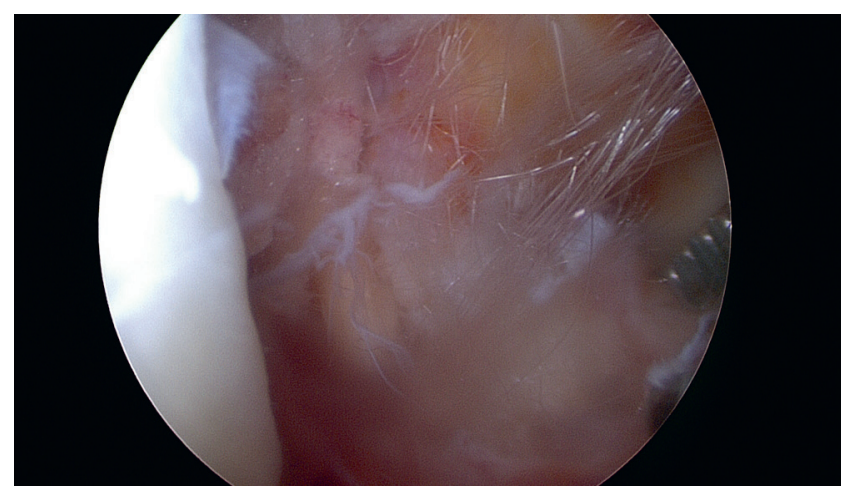

Fig. 7. Partial LARS rupture after 12 months - synthetic components

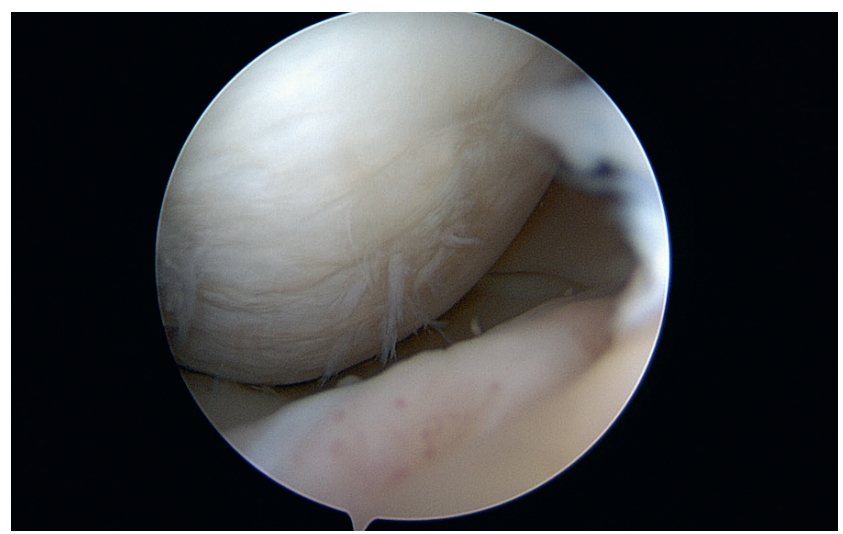

Fig. 8. Cartilage damage

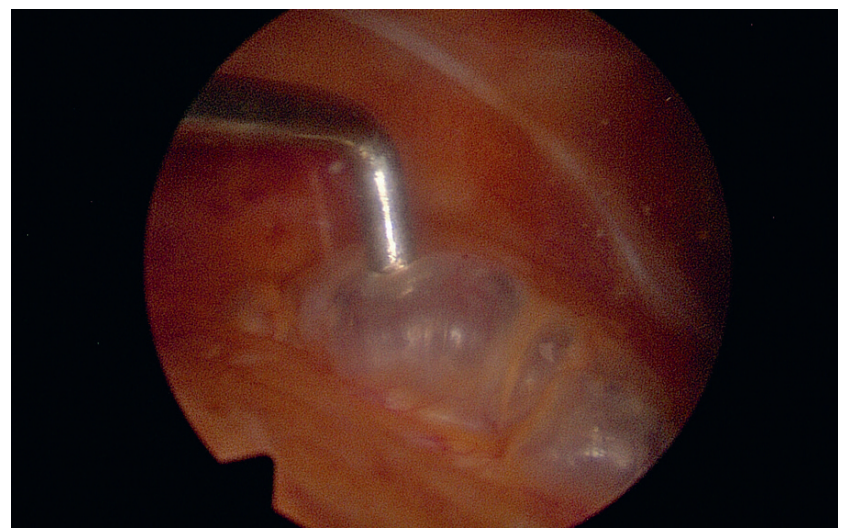

Fig. 9. Synovitis 


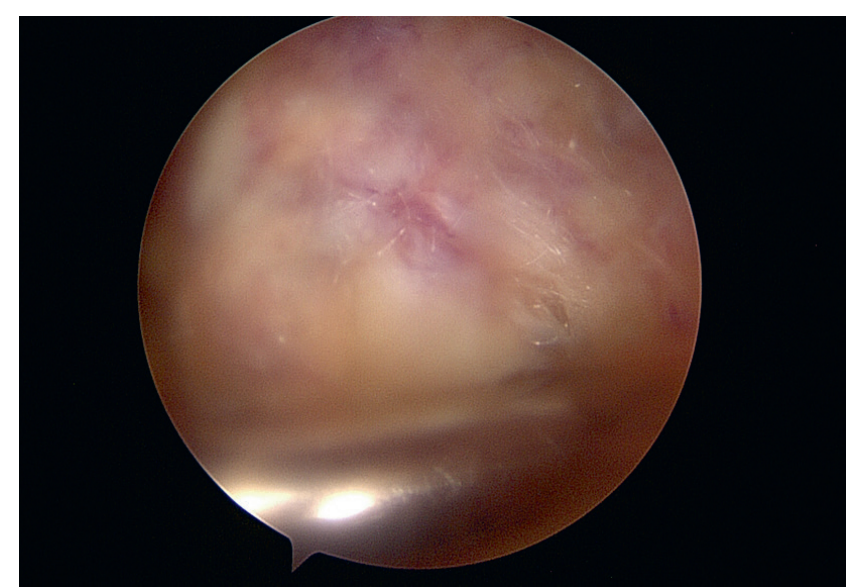

Fig. 10. Partial LARS rupture after 24 months - synthetic components

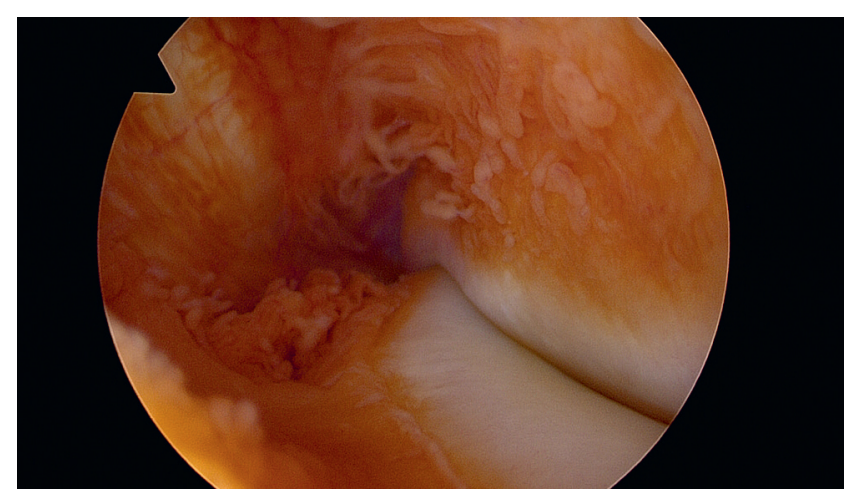

Fig. 11. Synovitis after 24 months

of increased load on the limb in a shorter time compared to natural grafts, increased mechanical strength, no local complications related to graft collection, and overall lower cost than autograft. ${ }^{23-26}$ This was also confirmed in our research. ${ }^{27-29}$

Despite the existence of the LARS technique since the 1990s, it is a rarely performed procedure compared to natural transplants. There are also few long-term postoperative follow-ups. ${ }^{25,26,30}$

The gold standard in ACL reconstruction is still reconstruction with the use of autogenous transplant. The LARS method is intended for people who require revision reconstructions, with limited possibilities of obtaining an autogenous transplant. It is not recommended for very young people. In our study, we also did not qualify such people for surgery. This technique is intended for elderly patients who want to quickly regain their fitness with the possibility of full load on the knee joint shortly after the procedure. ${ }^{31}$

One of the synthetic material grafts introduced in the $2^{\text {nd }}$ half of the $20^{\text {th }}$ century was a graft made of polyester, which evolved into a non-absorbable synthetic ligament device made of terephthalic polyethylene polyester fibers - LARS. ${ }^{32}$ It is intensively cleaned with the aim to remove potential machining residues and oils to further encourage soft tissue in-growth and reduce the risk of reactive synovitis. The LARS consists of 2 parts: an intra-articular part and an extra-articular part. The ligament intra-articular portion/scaffold is built of multiple parallel fibers that are twisted at $90^{\circ}$ angle. ${ }^{32}$ The part is composed of 2 longitudinal external rotation fibers without transverse fibers, being designed as an imitation of ACL anatomic structure. The extra-articular part is waved using longitudinal and transverse fibers with the aim to avoid ligament deformation. The short-term postoperative results of patients after ACL reconstruction with the use of LARS are very satisfying. ${ }^{33}$ A mean 2.5-year follow-up carried out by Dericks revealed encouraging results of treatment with the use of LARS. ${ }^{32}$ Also, a follow-up at a mean of 8 years reported by Parchi et al. showed satisfying results reflected in no postoperative complication occurrences and only 1 case of LARS rupture. ${ }^{34}$ On the other hand, the ten-year postoperative follow-up carried out by Tiefenboeck et al. revealed a lack of subjective satisfaction in $1 / 2$ of patients treated with the use of LARS; thus, the authors suggested not to consider the ligament system as a potential graft for primary reconstruction of the ACL and to treat it as an alternative graft in special cases. ${ }^{35}$ In our study, a very large number of revisions occurred after only 36 months.

Many studies have compared the results of ACL treatment using autografts and the synthetic LARS ligament. The most frequently compared autograft is the four-fold hamstring transplant. Patellar ligament (B-PT-B) and quadriceps tendon (QT) grafts have been studied less frequently. ${ }^{35-39}$

Zhong conducted a four-year follow-up study on a group of 60 patients (32 autografts and 28 synthetic grafts). The results were as follows: the difference in anterior tibia translation (ATT) of the operated and healthy limbs was on average $2.4 \pm 0.5 \mathrm{~mm}$ for the natural graft and $1.2 \pm 0.3 \mathrm{~mm}$ for the synthetic graft. A translation difference of more than $5 \mathrm{~mm}$ occurred in 3 patients operated on with hamstrings and in none in the LARS group. In terms of the IKDC 2000 rating, 87.5\% of the hamstrings group and $92.9 \%$ of the LARS group achieved a normal or near-normal result. The mean values of the Lysholm scale were $92.1 \pm 7.9$ and $94.6 \pm 9.2$, and for the Tegner scale they were $6.2 \pm 1.6$ and $6.6 \pm 1.8$, respectively, for autograft and synthetic graft group. In the $4 \mathrm{SGH}$ (hamstrings) group, $6.25 \%$ of respondents complained of a $5^{\circ}$ flexion deficit in the operated knee joint, and $3.13 \%$ suffered complications due to arthrofibrosis. In the LARS group, no complications related to limited ROM in the knee were observed. ${ }^{36}$ In terms of the IKDC 2000 and Lysholm scale, the results are similar to the results of our research.

Bianchi in his eight-year meta-analysis of the effectiveness of the 4SHG (25 patients) and LARS (25 patients) methods, assessed the occurrence of Lachman's symptom, which was $56 \%$ for LARS and $24 \%$ for 4 SHG, with the anterior drawer test at $68 \%$ for LARS and $16 \%$ for 4 SHG. ${ }^{37}$

Xiaoyu and Yecheng compared the effectiveness of treating ACL lesions using the technique of transplantation from the patellar tendon (BTB) and LARS in independent 
studies on groups of 62 patients (30 BTB and 32 LARS) and 50 patients (26 BTB and 24 LARS), with a four-year postoperative follow-up in both studies. ${ }^{38,39}$

All researchers, regardless of the type of material used as an autograft, draw the same conclusions: They emphasize significantly better ligamentous stability of the knee in physical examinations of LARS groups. We did not see a similar relationship. At the same time, the cited researchers describe the results of the IKDC 2000, Lysholm and Tegner tests, and subjective complaints about the knee joint without statistically significant differences in the compared groups. They write about significantly less pain and earlier stress on the limb in the LARS groups compared to the groups subjected to ACL reconstructions with the use of natural transplants. They note, however, that this effect is observed only in the early postoperative period up to 1 year (it is associated with the time of ligamentization - that is, reconstruction of natural grafts). ${ }^{36-40}$

Despite better results in the LARS treatment of ACL injuries, scientists agree that autograft augmentation remains the method of choice. They support this decision with a lack of documented long-term observations. The longest follow-up after the LARS procedure, which we found in the literature, was 11.6 years. ${ }^{24}$

In our opinion, such a large number of revision surgeries and complications do not justify the use of this method. To date, no published studies have directly compared the results of ACL reconstruction treatment with the LARS technique and allografts.

\section{Limitations}

The most obvious drawback of this study is the shortterm follow-up. In the future, studies involving long-term follow-up with patients that have undergone fully supervised physiotherapeutic procedures and comprehensive clinical and functional evaluations need to be taken into account.

\section{Conclusions}

Currently, numerous studies are being conducted that emphasize genetic predisposition to cruciate ligament injuries. The ACL "suture" techniques need additional testing and studies, and require much more scientific material. Research on the application of stem cells, scaffolds, plasma rich platelets, and xenografts are also breaking ground. These trends in the constant progression of ACL surgery will make way for a far more individualized method of operation and treatment.

Reconstruction of the ACL using a synthetic graft such as LARS gives similar results as autograft in a cohort follow-up. Long-term results show a large number of revision surgeries when using LARS. Using this method should be employed with caution.

\section{ORCID iDs}

Maciej Kentel (1D https://orcid.org/0000-0002-7610-5410 Michał Barnaś (D) https://orcid.org/0000-0003-2148-844X Jarosław Witkowski (D) https://orcid.org/0000-0002-2754-1339

Paweł Reichert (1) https://orcid.org/0000-0002-0271-4950

\section{References}

1. Anderson MJ, Browning WM III, Urband CE, Kluczynski MA, Bisson LJ. A systematic summary of systematic reviews on the topic of the anterior cruciate ligament. Orthop J Sports Med. 2016;4(3):232596711663 4074. doi:10.1177/2325967116634074

2. Prodromos CC, Han Y, Rogowski J, Joyce B, Shi K. A meta-analysis of the incidence of anterior cruciate ligament tears as a function of gender, sport, and a knee injury-reduction regimen. Arthroscopy. 2007;23(12):1320-1325.e6. doi:10.1016/j.arthro.2007.07.003

3. Ekstrand J. A 94\% return to elite level football after $A C L$ surgery: A proof of possibilities with optimal caretaking or a sign of knee abuse? Knee Surg Sports Traumatol Arthrosc. 2011;19(1):1-2. doi:10. 1007/s00167-010-1300-4

4. Czamara A, Królikowska A, Szuba Ł, Widuchowski W, Kentel M. Single- vs. double-bundle anterior cruciate ligament reconstruction: A new aspect of knee assessment during activities involving dynamic knee rotation. J Strength Cond Res. 2015;29(2):489-499. doi:10.1519/ JSC.0000000000000638

5. Królikowska A, Sikorski Ł, Czamara A, Reichert P. Effects of postoperative physiotherapy supervision duration on clinical outcome, speed, and agility in males 8 months after anterior cruciate ligament reconstruction. Med SciMonit. 2018;24:6823-6831. doi:10.12659/MSM.912162

6. Zeng C, Gao SG, Li H, et al. Autograft versus allograft in anterior cruciate ligament reconstruction: A meta-analysis of randomized controlled trials and systematic review of overlapping systematic reviews. Arthroscopy. 2016;32(1):153-163.e18. doi:10.1016/j.arthro.2015.07.027

7. Samuelsson K, Andersson D, Ahldén M, Fu FH, Musahl V, Karlsson J. Trends in surgeon preferences on anterior cruciate ligament reconstructive techniques. Clin Sports Med. 2013;32(1):111-126. doi:10.1016/ j.csm.2012.08.011

8. Buller LT, Best MJ, Baraga MG, Kaplan LD. Trends in anterior cruciate ligament reconstruction in the United States. Orthop J Sports Med. 2014;3(1):2325967114563664. doi:10.1177/2325967114563664

9. Chen H, Chen B, Tie K, Fu Z, Chen L. Single-bundle versus double-bundle autologous anterior cruciate ligament reconstruction: A metaanalysis of randomized controlled trials at 5-year minimum followup. J Orthop Surg Res. 2018;13(1):50. doi:10.1186/s13018-018-0753-x

10. Bieri KS, Scholz SM, Kohl S, Aghayev E, Staub LP. Dynamic intraligamentary stabilization versus conventional $A C L$ reconstruction: A matched study on return to work. Injury. 2017;48(6):1243-1248. doi:10.1016/j.injury.2017.03.004

11. Brown MJ, Carter T. ACL allograft: Advantages and when to use. Sports Med Arthrosc Rev. 2018;26(2):75-78. doi:10.1097/JSA.0000000000000194

12. Barrera Oro F, Sikka RS, Wolters B, et al. Autograft versus allograft: An economic cost comparison of anterior cruciate ligament reconstruction. Arthroscopy. 2011;27(9):1219-1225. doi:10.1016/j.arthro.2011.04.008

13. Cooper MT, Kaeding C. Comparison of the hospital cost of autograft versus allograft soft-tissue anterior cruciate ligament reconstructions. Arthroscopy. 2010;26(11):1478-1482. doi:10.1016/j.arthro. 2010.04.004

14. Debieux P, Franciozi $C E$, Lenza $M$, et al. Bioabsorbable versus metallic interference screws for graft fixation in anterior cruciate ligament reconstruction. Cochrane Database Syst Rev. 2016;7(7):CD009772. doi:10.1002/14651858.CD009772.pub2

15. Herz M. Die chirurgische Behandlung paralytischer SchlotterelenkeSeitenligamente oder Arthrodese? [The surgical treatmentof paralytic flail knees: Silk ligaments or arthrodesis]. Munch Med Wschr. 1906;51:2527-2529.

16. Schindler OS. Surgery for anterior cruciate ligament deficiency: A historical perspective. Knee Surg Sports Traumatol Arthrosc. 2012;20(1):547. doi:10.1007/s00167-011-1756-x

17. West RV, Harner CD. Graft selection in anterior cruciate ligament reconstruction. J Am Acad Orthop Surg. 2005;13(3):197-207. doi:10. 5435/00124635-200505000-00006 
18. Zoltan DJ, Reinecke C, Indelicato PA. Synthetic and allograft anterior cruciate ligament reconstruction. Clin Sports Med. 1988;7(4):773-784. PMID:3052882

19. Wilk RM, Richmond JC. Dacron ligament reconstruction for chronic anterior cruciate ligament insufficiency. Am J Sports Med. 1993;21(3): 374-380. doi:10.1177/036354659302100308

20. Maletius W, Gillquist J. Long-term results of anterior cruciate ligament reconstruction with a Dacron prosthesis: The frequency of osteoarthritis after seven to eleven years. Am J Sports Med. 1997;25(3):288-293. doi:10.1177/036354659702500303

21. Drogset JO, Grøntvedt T, Robak OR, Mølster A, Viset AT, Engebretsen L. A sixteen-year follow-up of three operative techniques for the treatment of acute ruptures of the anterior cruciate ligament. J Bone Joint Surg Am. 2006;88(5):944-952. doi:10.2106/JBJS.D.02876

22. Girgis FG, Marshall JL, Monajem A. The cruciate ligaments of the knee joint: Anatomical, functional and experimental analysis. Clin Orthop Relat Res. 1975;(106):216-231. doi:10.1097/00003086-197501000-00033

23. Cieślik-Bielecka A, Reichert P, Skowroński R, Królikowska A, Bielecki T. A new aspect of in vitro antimicrobial leukocyte- and platelet-rich plasma activity based on flow cytometry assessment. Platelets. 2019; 30(6):728-736. doi:10.1080/09537104.2018.1513472

24. Parchi PD, Ciapini G, Paglialunga $C$, et al. Anterior cruciate ligament reconstruction with LARS artificial ligament: Clinical results after a longterm follow-up. Joints. 2018;6(2):75-79. doi:10.1055/s-0038-1653950

25. Cerulli G, Antinolfi $P$, Bruè $S$, et al. Esperienza clinica nell'utilizzo di biomateriali nel ginocchio. GIOT. 2011;37(Suppl 1):159-166.

26. Jia Z, Xue C, Wang W, Liu T, Huang X, Xu W. Clinical outcomes of anterior cruciate ligament reconstruction using LARS artificial graft with an at least 7-year follow-up. Medicine (Baltimore). 2017;96(14):e6568. doi:10.1097/MD.0000000000006568

27. Czamara A, Markowska I, Królikowska A, Szopa A, Domagalska-Szopa M. Kinematics of rotation in joints of the lower limbs and pelvis during gait: Early result of SB ACLR approach versus DB ACLR approach. Biomed Res Int. 2015;2015:707168. doi:10.1155/2015/707168

28. Królikowska A, Reichert P, Czamara A, Krzemińska K. Peak torque angle of anterior cruciate ligament-reconstructed knee flexor muscles in patients with semitendinosus and gracilis autograft is shifted towards extension regardless of the postoperative duration of supervised physiotherapy. PLoS One. 2019;14(2):e0211825. doi:10.1371/journal. pone. 0211825

29. Królikowska A, Sikorski Ł, Czamara A, Reichert P. Are the knee extensor and flexor muscles isokinetic parameters affected by the duration of postoperative physiotherapy supervision in patients eight months after $A C L$ reconstruction with the use of semitendinosus and gracilis tendons autograft? Acta Bioeng Biomech. 2018;20(4):89-100.
30. Wang $\mathrm{CL}$, Hsiao CK, Ku MC, Chang CH. Arthroscopic anterior cruciate ligament reconstruction with LARS artificial ligament: An 8-15year follow-up. J Mech Med Biol. 2013;13(2):1350046.

31. Seil R, Weitz FK, Pape D. Surgical-experimental principles of anterior cruciate ligament $(A C L)$ reconstruction with open growth plates. J Exp Orthop. 2015;2(1):11. doi:10.1186/s40634-015-0027-z

32. Dericks G. Ligament advanced reinforcement system anterior cruciate ligament reconstruction. Oper Tech Sports Med. 1995;3(3):187205. doi:10.1016/S1060-1872(95)80009-3

33. Jia ZY, Zhang C, Cao SQ, et al. Comparison of artificial graft versus autograft in anterior cruciate ligament reconstruction: A meta-analysis. BMC Musculoskelet Disord. 2017;18(1):309. doi:10.1186/s12891-017-1672-4

34. Parchi PD, Gianluca C, Dolfi L, et al. Anterior cruciate ligament reconstruction with $\mathrm{LARS}^{\mathrm{TM}}$ artificial ligament results at a mean follow-up of eight years. Int Orthop. 2013;37(8):1567-1574. doi:10.1007/s00264013-1917-2

35. Tiefenboeck TM, Thurmaier E, Tiefenboeck MM, et al. Clinical and functional outcome after anterior cruciate ligament reconstruction using the LARS ${ }^{\mathrm{TM}}$ system at a minimum follow-up of 10 years. Knee. 2015;22(6):565-568. doi:10.1016/j.knee.2015.06.003

36. Liu ZT, Zhang $X L$, Jiang $Y$, Zeng BF. Four-strand hamstring tendon autograft versus LARS artificial ligament for anterior cruciate ligament reconstruction. Int Orthop. 2010;34(1):45-49. doi:10.1007/s00 264-009-0768-3

37. Bianchi N, Sacchetti F, Bottai $\mathrm{V}$, et al. LARS versus hamstring tendon autograft in anterior cruciate ligament reconstruction: A single-centre, single surgeon retrospective study with 8 years of follow-up. Eur J Orthop Surg Traumatol. 2019;29(2):447-453. doi:10.1007/s00590018-2304-X

38. Li Y, Zhang W, Wu Y, et al. A comparison of effectiveness between ligament advanced reinforcement system and bone-patellar tendonbone autograft for anterior cruciate ligament reconstruction [in Chinese]. Zhongguo Xiu Fu Chong Jian Wai Ke Za Zhi. 2012;26(9):1045-1050.

39. Pan X, Wen H, Wang L, Ge T. Bone-patellar tendon-bone autograft versus LARS artificial ligament for anterior cruciate ligament reconstruction. Eur J Orthop Surg Traumatol. 2013;23(7):819-823. doi:10. 1007/s00590-012-1073-1

40. Lavoie P, Fletcher J, Duval N. Patient satisfaction needs as related to knee stability and objective findings after $A C L$ reconstruction using the LARS artificial ligament. Knee. 2000;7(3):157-163. doi:10. 1016/s0968-0160(00)00039-9 